\title{
Existence of concave symmetric positive solutions for a three-point boundary value problems
}

\author{
Ummahan Akcan ${ }^{1}$ and Nuket $\mathrm{A} \mathrm{Hamal}{ }^{2 *}$
}

\section{"Correspondence:}

nuket.aykut@ege.edu.tr

${ }^{2}$ Department of Mathematics, Ege

University, Bornova, Izmir, 35100,

Turkey

Full list of author information is

available at the end of the article

\begin{abstract}
In this paper, we investigate the existence of triple concave symmetric positive solutions for the nonlinear boundary value problems with integral boundary conditions. The proof is based upon the Avery and Peterson fixed point theorem. An example which supports our theoretical result is also indicated.
\end{abstract}

MSC: 34B10; 39B18; 39A10

Keywords: three-point boundary value problem; symmetric positive solution; integral boundary condition; fixed point theorem

\section{Introduction}

Consider the boundary value problem (BVP)

$$
\left\{\begin{array}{l}
u^{\prime \prime}(x)+f\left(x, u(x), u^{\prime}(x)\right)=0, \quad x \in(0,1) \\
u(0)=u(1)=\alpha \int_{0}^{\eta} u(s) d s
\end{array}\right.
$$

where $f:(0,1) \times[0, \infty) \times \mathbb{R} \rightarrow[0, \infty)$ is continuous and $\alpha, \eta \in(0,1)$. A function $u \in \mathcal{C}[0,1]$ is said to be symmetric on $[0,1]$ if

$$
u(x)=u(1-x), \quad x \in[0,1]
$$

By a symmetric positive solution of BVP (1.1) we mean a symmetric function $u \in \mathcal{C}^{2}[0,1]$ such that $u(x) \geq 0$ for $x \in(0,1)$ and $u(x)$ satisfies BVP $(1.1)$.

Recently, many authors have focused on the existence of symmetric positive solutions for ordinary differential equation boundary value problems; for example, see [1-5] and the references therein. However, multi-point boundary value problems included the most recent works $[1-4,6-9]$ and boundary value problems with integral boundary conditions for ordinary differential equations have been studied by many authors; one may refer to [ 5 , 10-12]. Motivated by the works mentioned above, we aim to investigate existence results for concave symmetric positive solutions of BVP (1.1) by applying the fixed point theorem of Avery and Peterson.

The organization of this paper is as follows. Section 2 of this paper contains some preliminary lemmas. In Section 3, by applying the Avery and Peterson fixed point theorem, 
we obtain concave symmetric positive solutions for BVP (1.1). In Section 4, an example will be presented to illustrate the applicability of our result.

Throughout this paper, we always assume that the following assumption is satisfied:

(H1) $f \in \mathcal{C}((0,1) \times[0,+\infty) \times \mathbb{R},[0,+\infty)), f(x, u, v)=f(1-x, u,-v)$ for $x \in\left(0, \frac{1}{2}\right]$, and $f(x, u, v) \geq 0$ for all $(x, u, v) \in(0,1) \times[0,+\infty) \times \mathbb{R}$.

\section{Preliminaries}

In this section, we present several lemmas that will be used in the proof of our result.

Lemma 2.1 Let $h \in \mathcal{C}[0,1]$ and $\alpha \eta \neq 1$, then the $B V P$

$$
\begin{aligned}
& u^{\prime \prime}(x)+h(x)=0, \quad 0<x<1, \\
& u(0)=u(1)=\alpha \int_{0}^{\eta} u(s) d s,
\end{aligned}
$$

has a solution

$$
u(x)=\int_{0}^{1}(H(s)+G(x, s)) h(s) d s,
$$

where

$$
\begin{aligned}
& V(s)=\left\{\begin{array}{ll}
(\eta-s)^{2}, & s \leq \eta, \\
0, & \eta \leq s,
\end{array} \quad H(s)=\frac{\alpha \eta^{2}}{2(1-\alpha \eta)}(1-s)-\frac{\alpha}{2(1-\alpha \eta)} V(s),\right. \\
& G(x, s)= \begin{cases}s(1-x), & 0 \leq s \leq x \leq 1 \\
x(1-s), & 0 \leq x \leq s \leq 1 .\end{cases}
\end{aligned}
$$

Proof Suppose that $u \in \mathcal{C}^{2}[0,1]$ is a solution of problem (2.1) and (2.2). Then we have

$$
u^{\prime \prime}(x)=-h(x) .
$$

For $x \in[0,1]$, by integration from 0 to 1 , we have

$$
u^{\prime}(x)=u^{\prime}(0)-\int_{0}^{x} h(s) d s .
$$

For $x \in[0,1]$, by integration again from 0 to 1 , we have

$$
u(x)=u^{\prime}(0) x-\int_{0}^{x}\left(\int_{0}^{\tau} h(s) d s\right) d \tau .
$$

That is,

$$
u(x)=u(0)+u^{\prime}(0) x-\int_{0}^{x}(x-s) h(s) d s,
$$

therefore,

$$
u(1)=u(0)+u^{\prime}(0)-\int_{0}^{1}(1-s) h(s) d s .
$$


From condition (2.2), we have

$$
u^{\prime}(0)=\int_{0}^{1}(1-s) h(s) d s
$$

Integrating (2.6) from 0 to $\eta$, where $\eta \in(0,1)$, we have

$$
\begin{aligned}
\int_{0}^{\eta} u(s) d s & =u(0) \eta+u^{\prime}(0) \frac{\eta^{2}}{2}-\int_{0}^{\eta}\left(\int_{0}^{\tau}(\tau-s) h(s) d s\right) d \tau \\
& =u(0) \eta+u^{\prime}(0) \frac{\eta^{2}}{2}-\frac{1}{2} \int_{0}^{\eta}(\eta-s)^{2} h(s) d s
\end{aligned}
$$

and from $u(0)=\alpha \int_{0}^{\eta} u(s) d s$, we have

$$
u(0)=\frac{\alpha \eta^{2}}{2(1-\alpha \eta)} u^{\prime}(0)-\frac{\alpha}{2(1-\alpha \eta)} \int_{0}^{\eta}(\eta-s)^{2} h(s) d s
$$

therefore, (2.1) and (2.2) have a unique solution

$$
\begin{aligned}
u(x)= & \frac{\alpha \eta^{2}}{2(1-\alpha \eta)} \int_{0}^{1}(1-s) h(s) d s-\frac{\alpha}{2(1-\alpha \eta)} \int_{0}^{\eta}(\eta-s)^{2} h(s) d s \\
& +x \int_{0}^{1}(1-s) h(s) d s-\int_{0}^{x}(x-s) h(s) d s .
\end{aligned}
$$

From (2.4) and (2.5), we obtain

$$
u(x)=\int_{0}^{1}(H(s)+G(x, s)) h(s) d s
$$

The proof is complete.

The functions $H$ and $G$ have the following properties.

Lemma 2.2 If $\alpha, \eta \in(0,1)$, then we have

$$
H(s) \geq 0 \quad \text { for } s \in[0,1] .
$$

Proof From the definition of $H(s), s \in(0,1)$, and $\alpha, \eta \in(0,1)$, we have $H(s) \geq 0$.

Lemma $2.3 G(1-x, 1-s)=G(x, s), 0 \leq G(x, s) \leq G(s, s)$ for $x, s \in[0,1]$.

Proof From the definition of $G(x, s)$, we get $G(1-x, 1-s)=G(x, s)$ and $0 \leq G(x, s) \leq G(s, s)$ for $x, s \in[0,1]$.

Lemma 2.4 If $f\left(x, u(x), u^{\prime}(x)\right) \in \mathcal{C}((0,1) \times[0,+\infty) \times \mathbb{R},[0,+\infty))$ and we let $\alpha, \eta \in(0,1)$, then the unique solution $u$ of $B V P(1.1)$ satisfies $u(x) \geq 0$ for $x \in[0,1]$.

Proof From the definition of $u(x)$, Lemma 2.2, Lemma 2.3, and $f\left(x, u(x), u^{\prime}(x)\right) \in \mathcal{C}((0,1) \times$ $[0,+\infty) \times \mathbb{R},[0,+\infty))$, we have $u(x) \geq 0$. 
Let $E=\mathcal{C}^{2}[0,1]$. Then $E$ is Banach space with the norm $\|u\|=\max \left\{\|u\|_{\infty},\left\|u^{\prime}\right\|_{\infty}\right\}$, where $\|u\|_{\infty}=\max _{x \in[0,1]}|u(x)|$.

We define the cone $P \subset E$ by

$$
P=\{u \in E: u(x) \geq 0 \text { is concave and } u(x)=u(1-x), x \in[0,1]\} \text {. }
$$

Define the operator $T: P \rightarrow E$ as follows:

$$
(T u)(x)=\int_{0}^{1}(H(s)+G(x, s)) f\left(s, u(s), u^{\prime}(s)\right) d s \quad \text { for } x \in[0,1],
$$

where $G(x, s)$ and $H(s)$ are given by (2.4) and (2.5). Clearly, $u$ is the solution of BVP (1.1) if and only if $u$ is a fixed point of the operator $T$.

Lemma 2.5 Let $0<\alpha<1$ and $0<\eta<1$. For $u \in P$, there exists a real number $M>0$ such that

$$
\max _{0 \leq x \leq 1}|u(x)| \leq M \max _{0 \leq x \leq 1}\left|u^{\prime}(x)\right|
$$

where $M=\frac{1}{2(1-\alpha \eta)}$.

Proof For any $u \in P$, we have

$$
\max _{0 \leq x \leq 1}|u(x)|=u\left(\frac{1}{2}\right), \quad \max _{0 \leq x \leq 1}\left|u^{\prime}(x)\right|=u^{\prime}(0),
$$

the concavity of $u$ implies that

$$
u^{\prime}(0) \geq \frac{u\left(\frac{1}{2}\right)-u(0)}{\frac{1}{2}},
$$

then

$$
u\left(\frac{1}{2}\right) \leq \frac{u^{\prime}(0)}{2}+u(0)
$$

Now we divide the proof into two cases.

Case 1 . If $0<\eta \leq \frac{1}{2}$, then the concavity of $u$ implies that

$$
\int_{0}^{\eta} u(s) d s \leq \eta u(\eta)
$$

where $\eta u(\eta)$ is the area of rectangle, then

$$
u(0) \leq \alpha \eta u(\eta) \leq \alpha \eta u\left(\frac{1}{2}\right) .
$$

So

$$
u(0) \leq \alpha \eta u\left(\frac{1}{2}\right),
$$


then from (2.8) and (2.9), we have

$$
u\left(\frac{1}{2}\right) \leq \frac{1}{2(1-\alpha \eta)} u^{\prime}(0)
$$

that is,

$$
\max _{0 \leq x \leq 1}|u(x)| \leq \frac{1}{2(1-\alpha \eta)} \max _{0 \leq x \leq 1}\left|u^{\prime}(x)\right| .
$$

Case 2. If $\frac{1}{2} \leq \eta<1$, then the concavity and symmetry of $u$ imply that

$$
\int_{0}^{\eta} u(s) d s \leq \int_{0}^{1} u(s) d s=2 \int_{0}^{\frac{1}{2}} u(s) d s \leq 2 u\left(\frac{1}{2}\right) \frac{1}{2} .
$$

So

$$
u(0) \leq \alpha u\left(\frac{1}{2}\right)
$$

then from (2.8) and (2.10), we have

$$
u\left(\frac{1}{2}\right) \leq \frac{1}{2(1-\alpha)} u^{\prime}(0)
$$

that is,

$$
\max _{0 \leq x \leq 1}|u(x)| \leq \frac{1}{2(1-\alpha)} \max _{0 \leq x \leq 1}\left|u^{\prime}(x)\right|,
$$

where $M=\min \left\{\frac{1}{2(1-\alpha \eta)}, \frac{1}{2(1-\alpha)}\right\}=\frac{1}{2(1-\alpha \eta)}$, these equations complete the proof.

Lemma 2.6 Let $0<\alpha<1$ and $0<\eta<1$. For $u \in P$, there exists a real number $0<N<1$ such that

$$
\min _{0 \leq x \leq 1}|u(x)| \geq N\|u\|_{\infty}
$$

where $N=\max \left\{\frac{\alpha \eta^{2}}{\alpha \eta^{2}-\alpha \eta+1}, \frac{\alpha}{4-\alpha}\right\}$.

Proof For any $u \in P$, we have

$$
\min _{0 \leq x \leq 1}|u(x)|=u(0)=u(1), \quad\|u\|_{\infty}=\max _{0 \leq x \leq 1}|u(x)|=u\left(\frac{1}{2}\right)
$$

and because the graph of $u$ is concave, we have

$$
\int_{0}^{\eta} u(s) d s \geq \frac{\eta}{2}(u(\eta)+u(0))
$$

then

$$
-u(\eta) \geq \frac{\alpha \eta-2}{\alpha \eta} u(0)
$$

Now we divide the proof into two cases. 
Case 1 . If $0<\eta \leq \frac{1}{2}$, then using concavity and positivity of $u$, we get

$$
\frac{u(\eta)-u(0)}{\eta} \geq \frac{u\left(\frac{1}{2}\right)-u(0)}{\frac{1}{2}}
$$

So

$$
\frac{2 \eta-1}{\eta} u(0) \geq 2 u\left(\frac{1}{2}\right)-\frac{1}{\eta} u(\eta),
$$

then from (2.11) and (2.12), we have

$$
u(0) \geq \frac{\alpha \eta^{2}}{\alpha \eta^{2}-\alpha \eta+1} u\left(\frac{1}{2}\right)
$$

that is

$$
\min _{0 \leq x \leq 1}|u(x)| \geq \frac{\alpha \eta^{2}}{\alpha \eta^{2}-\alpha \eta+1}\|u\|_{\infty} .
$$

Case 2. If $\frac{1}{2} \leq \eta<1$, then using concavity and positivity of $u$, we get

$$
\int_{0}^{\eta} u(s) d s \geq \frac{\left(u\left(\frac{1}{2}\right)-u(0)\right) \frac{1}{2}}{2}+\frac{u(0)}{2}
$$

So

$$
u(0) \geq \frac{\alpha}{4-\alpha} u\left(\frac{1}{2}\right)
$$

that is,

$$
\min _{0 \leq x \leq 1}|u(x)| \geq \frac{\alpha}{4-\alpha}\|u\|_{\infty}
$$

where $N=\max \left\{\frac{\alpha \eta^{2}}{\alpha \eta^{2}-\alpha \eta+1}, \frac{\alpha}{4-\alpha}\right\}$, and for $\alpha, \eta \in(0,1)$, one can easily see that $N \in(0,1)$. These equations complete the proof.

\section{Existence of triple concave symmetric positive solutions for BVP (1.1)}

In this section, we will apply the Avery-Peterson fixed point theorem to establish the existence of at least three concave symmetric positive solutions of BVP (1.1).

Let $\alpha, \gamma, \theta, \psi$ be maps on $P$ with $\alpha$ a nonnegative continuous concave functional; $\gamma, \theta$ nonnegative continuous convex functionals, and $\psi$ a nonnegative continuous functional. Then for positive numbers $a, b, c, d$ we define the following subsets of $P$ :

$$
\begin{aligned}
& P(\gamma, d)=\{u \in P \mid \gamma(u)<d\}, \\
& P(\alpha, \gamma, b, d)=\{u \in \overline{P(\gamma, d)} \mid \alpha(u) \geq b\}, \\
& P(\alpha, \theta, \gamma, b, c, d)=\{u \in \overline{P(\gamma, d)} \mid \alpha(u) \geq b, \theta(u) \leq c\}, \\
& R(\psi, \gamma, a, d)=\{u \in \overline{P(\gamma, d)} \mid \psi(u) \geq a\} .
\end{aligned}
$$


Now we state the Avery-Peterson fixed point theorem.

Theorem 3.1 $([2,3,9,13])$ Let $P$ be cone in Banach space E. Let $\gamma$ and $\theta$ be nonnegative continuous convex functionals on $P, \alpha$ be a nonnegative continuous concave functional on $P$, and $\psi$ be a nonnegative continuous functional on P leading to

$$
\psi(\lambda u) \leq \lambda \psi(u) \text { for all } 0 \leq \lambda \leq 1
$$

and

$$
\alpha(u) \leq \psi(u), \quad\|u\| \leq M \gamma(u) \text { for all } u \in \overline{P(\gamma, d)} \text { with } M, d \text { positive numbers. }
$$

Suppose $T: P \rightarrow P$ is completely continuous and there exist positive numbers $a, b, c$ with $a<b$ such that

$\left(\mathrm{S}_{1}\right): \quad\{u \in P(\alpha, \theta, \gamma, b, c, d) \mid \alpha(u)>b\} \neq \emptyset \quad$ and

$$
\alpha(T u)>b \quad \text { for } u \in P(\alpha, \theta, \gamma, b, c, d) \text {; }
$$

$\left(\mathrm{S}_{2}\right): \quad \alpha(T u)>b \quad$ for $u \in P(\alpha, \gamma, b, d)$ with $\theta(T u)>c ;$

$\left(\mathrm{S}_{3}\right): \quad 0 \notin R(\psi, \gamma, a, d)$ and $\psi(T u)<a \quad$ for $u \in R(\psi, \gamma, a, d)$ with $\psi(u)=a$.

Then $T$ has at least three fixed points $u_{1}, u_{2}, u_{3} \in \overline{P(\gamma, d)}$ such that

$$
\begin{aligned}
& \gamma\left(u_{i}\right) \leq d \quad \text { for } i=1,2,3 ; \quad \psi\left(u_{1}\right)<a ; \\
& \psi\left(u_{2}\right)>a \quad \text { with } \alpha\left(u_{2}\right)<b ; \quad \alpha\left(u_{3}\right)>b .
\end{aligned}
$$

Lemma 3.1 Assume that $(\mathrm{H} 1)$ is satisfied and let $\alpha \eta \in(0,1)$. Then the operator $T$ is completely continuous.

Proof For any $u \in P$, from the expression of $T u$, we know

$$
\left\{\begin{array}{l}
(T u)^{\prime \prime}(x)+f\left(x, u(x), u^{\prime}(x)\right)=0, \quad x \in(0,1) \\
(T u)(0)=(T u)(1)=\alpha \int_{0}^{\eta}(T u)(s) d s
\end{array}\right.
$$

Clearly, $T u$ is concave. From the definition of $T u$, Lemma 2.2, and Lemma 2.3 we see that $T u$ is nonnegative on $[0,1]$. We now show that $T u$ is symmetric about $\frac{1}{2}$. From Lemma 2.3 and (H1), for $x \in[0,1]$, we have

$$
\begin{aligned}
(T u)(1-x) & =\int_{0}^{1}(H(s)+G(1-x, s)) f\left(s, u(s), u^{\prime}(s)\right) d s \\
& =\int_{0}^{1} H(s) f\left(s, u(s), u^{\prime}(s)\right) d s+\int_{0}^{1} G(1-x, s) f\left(s, u(s), u^{\prime}(s)\right) d s \\
& =\int_{0}^{1} H(s) f\left(s, u(s), u^{\prime}(s)\right) d s-\int_{1}^{0} G(1-x, 1-s) f\left(1-s, u(1-s), u^{\prime}(1-s)\right) d s \\
& =\int_{0}^{1} H(s) f\left(s, u(s), u^{\prime}(s)\right) d s+\int_{0}^{1} G(x, s) f\left(1-s, u(s),-u^{\prime}(s)\right) d s
\end{aligned}
$$




$$
\begin{aligned}
& =\int_{0}^{1} H(s) f\left(s, u(s), u^{\prime}(s)\right) d s+\int_{0}^{1} G(x, s) f\left(s, u(s), u^{\prime}(s)\right) d s \\
& =\int_{0}^{1}(H(s)+G(x, s)) f\left(s, u(s), u^{\prime}(s)\right) d s \\
& =(T u)(x)
\end{aligned}
$$

therefore, $T P \subset P$.

The continuity of $T$ with respect to $u(x) \in \mathcal{C}^{2}[0,1]$ is clear. We now show that $T$ is compact. Suppose that $D \subset P$ is a bounded set. Then there exists $r$ such that

$$
D=\{u \in P \mid\|u\| \leq r\} .
$$

For any $u \in D$, we have

$$
0 \leq f\left(s, u(s), u^{\prime}(s)\right) \leq \max \left\{f\left(s, u, u^{\prime}\right) \mid s \in[0,1], u \in[0, r], u^{\prime} \in[-r, r]\right\}=: M
$$

So, we have from $(2.7)$

$$
\begin{aligned}
\|(T u)(x)\|_{\infty} & =\max _{x \in[0,1]}\left|\int_{0}^{1}(H(s)+G(x, s)) f\left(s, u(s), u^{\prime}(s)\right) d s\right| \\
& \leq M \int_{0}^{1} H(s) d s+M \max _{x \in[0,1]} \int_{0}^{1} G(x, s) d s=: L
\end{aligned}
$$

and

$$
\begin{aligned}
\left\|(T u)^{\prime}(x)\right\|_{\infty} & =\max _{x \in[0,1]}\left|\int_{0}^{1}(1-s) f\left(s, u(s), u^{\prime}(s)\right) d s-\int_{0}^{x} f\left(s, u(s), u^{\prime}(s)\right) d s\right| \\
& \leq \frac{M}{2}+M
\end{aligned}
$$

These equations imply that the operator $T$ is uniformly bounded. Now we show that $T u$ is equi-continuous.

We separate these three conditions:

Case (i). $0 \leq x_{1} \leq x_{2} \leq \frac{1}{2}$;

Case (ii). $\frac{1}{2} \leq x_{1} \leq x_{2} \leq 1$;

Case (iii). $0 \leq x_{1} \leq \frac{1}{2} \leq x_{2} \leq 1$.

We solely need to deal with Case (i) since the proofs of the other two are analogous. For $0 \leq x_{1} \leq x_{2} \leq \frac{1}{2}$, we have

$$
\begin{aligned}
& \left|(T u)\left(x_{2}\right)-(T u)\left(x_{1}\right)\right| \\
& \quad=\left|\int_{0}^{1}\left(G\left(x_{2}, s\right)-G\left(x_{1}, s\right)\right) f\left(s, u(s), u^{\prime}(s)\right) d s\right| \\
& \quad \leq \begin{cases}\int_{0}^{1}\left|\left(x_{2}-x_{1}\right)(1-s)\right| f\left(s, u(s), u^{\prime}(s)\right) d s, & 0 \leq x_{1} \leq x_{2} \leq s \leq \frac{1}{2} \\
\int_{0}^{1}\left|s\left(x_{1}-x_{2}\right)\right| f\left(s, u(s), u^{\prime}(s)\right) d s, & 0 \leq s \leq x_{1} \leq x_{2} \leq \frac{1}{2} \\
\int_{0}^{1}\left|s\left(1-x_{2}\right)-x_{1}(1-s)\right| f\left(s, u(s), u^{\prime}(s)\right) d s, & 0 \leq x_{1} \leq s \leq x_{2} \leq \frac{1}{2}\end{cases} \\
& \quad \leq\left\{\begin{array}{l}
\frac{M}{2}\left|x_{2}-x_{1}\right|, \\
\frac{M}{2}\left|x_{2}-x_{1}\right|, \\
\frac{3 M}{2}\left|x_{2}-x_{1}\right| .
\end{array}\right.
\end{aligned}
$$


In addition

$$
\left|(T u)^{\prime}\left(x_{2}\right)-(T u)^{\prime}\left(x_{1}\right)\right|=\left|\int_{x_{2}}^{x_{1}} f\left(s, u(s), u^{\prime}(s)\right) d s\right| \leq M\left|x_{2}-x_{1}\right| .
$$

So, we see that $T u$ is equi-continuous. By applying the Arzela-Ascoli theorem, we can guarantee that $T(D)$ is relatively compact, which means $T$ is compact. Then we find that $T$ is completely continuous. This completes the proof.

For convenience, we denote

$$
\begin{aligned}
& L=\int_{0}^{1}\left(H(s)+G\left(\frac{1}{2}, s\right)\right) d s, \\
& N=\max \left\{\frac{\alpha \eta^{2}}{\alpha \eta^{2}-\alpha \eta+1}, \frac{\alpha}{4-\alpha}\right\}, \\
& M=\frac{1}{2(1-\alpha \eta)}, \\
& \delta=\int_{0}^{1} H(s) d s .
\end{aligned}
$$

Theorem 3.2 Suppose (H1) holds and let $\alpha, \eta \in(0,1)$. Moreover, there exist nonnegative numbers $0<a<b \leq 2 d \delta$ such that

(B $\left.\mathrm{B}_{1}\right) \quad f(x, u, v) \leq 2 d \quad$ for $(x, u, v) \in[0,1] \times[0, M d] \times[-d, d]$;

(B) $f(x, u, v)>\frac{b}{\delta} \quad$ for $(x, u, v) \in[0,1] \times\left[b, \frac{b}{N}\right] \times[-d, d]$;

(B) $f(x, u, v)<\frac{a}{L} \quad$ for $(x, u, v) \in[0,1] \times[0, a] \times[-d, d]$,

then BVP (1.1) has at least three concave symmetric positive solutions $u_{1}, u_{2}, u_{3}$ such that

$$
\begin{aligned}
& \max _{0 \leq x \leq 1}\left|u_{i}^{\prime}(x)\right| \leq d \quad \text { for } i=1,2,3 ; \quad \max _{0 \leq x \leq 1}\left|u_{1}(x)\right|<a, \\
& \max _{0 \leq x \leq 1}\left|u_{2}(x)\right|>a \quad \text { with } \min _{0 \leq x \leq 1}\left|u_{2}(x)\right|<b, \quad \min _{0 \leq x \leq 1}\left|u_{3}(x)\right|>b .
\end{aligned}
$$

Proof BVP (1.1) has a solution $u=u(x)$ if and only if $u$ solves the operator equation $u=T(u)$. So we need to verify that operator $T$ satisfies the Avery-Peterson fixed point theorem, which will prove the existence of at least three fixed points of $T$.

Complete continuity of $T$ is clear from Lemma 3.1. Define the nonnegative functionals $\alpha, \theta, \gamma$, and $\psi$ by

$$
\gamma(u)=\max _{0 \leq x \leq 1}\left|u^{\prime}(x)\right|, \quad \psi(u)=\theta(u)=\max _{0 \leq x \leq 1}|u(x)|, \quad \alpha(u)=\min _{0 \leq x \leq 1}|u(x)| .
$$

Then in the cone $P, \theta$ and $\gamma$ are convex as $\alpha$ is concave. It is well known that $\psi(\lambda u) \leq \lambda \psi(u)$ for all $0 \leq \lambda \leq 1$ and $\alpha(u) \leq \psi(u)$. Moreover, from Lemma 2.5, $\|u\| \leq M \gamma(u)$. Now, we will prove the main theorem in four steps.

Step 1. We will show that $T: \overline{P(\gamma, d)} \rightarrow \overline{P(\gamma, d)}$. 
If $u \in \overline{P(\gamma, d)}$, then $\gamma(u)=\max _{0 \leq x \leq 1}\left|u^{\prime}(x)\right| \leq d$. Lemma 2.5 yields $\max _{0 \leq x \leq 1}|u(x)| \leq$ $M d$, then the condition $\left(\mathrm{B}_{1}\right)$ implies that $f(x, u, v) \leq 2 d$. On the other hand, for any $u \in P$, there is $T(u) \in P$, then $T(u)$ is concave, symmetric, and positive on $[0,1]$ and $\max _{0 \leq x \leq 1}\left|(T u)^{\prime}(x)\right|=\left|(T u)^{\prime}(0)\right|$, and we have

$$
\begin{aligned}
\gamma(T u) & =\max _{0 \leq x \leq 1}\left|(T u)^{\prime}(x)\right|=\left|(T u)^{\prime}(0)\right| \\
& =\int_{0}^{1}(1-s) f\left(s, u(s), u^{\prime}(s)\right) d s \leq 2 d \cdot \frac{1}{2}=d .
\end{aligned}
$$

Then $(T u) \in \overline{P(\gamma, d)}$. Therefore $T: \overline{P(\gamma, d)} \rightarrow \overline{P(\gamma, d)}$.

Step 2 . To check if condition $\left(\mathrm{S}_{1}\right)$ of Theorem 3.1 is satisfied, we choose $u(x)=\frac{b}{N}$. Clearly,

$$
\begin{aligned}
& \alpha(u)=\min _{0 \leq x \leq 1}|u(x)|=u(0)=\frac{b}{N}>b, \\
& \theta(u)=\max _{0 \leq x \leq 1}|u(x)|=u\left(\frac{1}{2}\right)=\frac{b}{N}, \\
& \gamma(u)=\max _{0 \leq x \leq 1}\left|u^{\prime}(x)\right|=\left|u^{\prime}(0)\right|=0 \leq d .
\end{aligned}
$$

Thus, $u \in P\left(\alpha, \theta, \gamma, b, \frac{b}{N}, d\right)$ and $\left\{u \in P\left(\alpha, \theta, \gamma, b, \frac{b}{N}, d\right) \mid \alpha(u)>b\right\} \neq \emptyset$.

If $u \in P\left(\alpha, \theta, \gamma, b, \frac{b}{N}, d\right)$, then we have $b \leq u(x) \leq \frac{b}{N},-d \leq u^{\prime}(x) \leq d$. From condition $\left(\mathrm{B}_{2}\right)$, we have $f\left(x, u(x), u^{\prime}(x)\right)>\frac{b}{\delta}$, and it follows that

$$
\begin{aligned}
\alpha(T(u)) & =\min _{0 \leq x \leq 1}|T u(x)|=(T u)(0) \\
& =\int_{0}^{1}(H(s)+G(0, s)) f\left(s, u(s), u^{\prime}(s)\right) d s \\
& >\frac{b}{\delta} \int_{0}^{1} H(s) d s=b .
\end{aligned}
$$

This shows that condition $\left(\mathrm{S}_{1}\right)$ of Theorem 3.1 is satisfied.

Step 3. We will show that condition $\left(\mathrm{S}_{2}\right)$ of Theorem 3.1 is satisfied. Take $u \in P(\alpha, \gamma, b, d)$ with $\theta(T u)>\frac{b}{N}$. Then from Lemma 2.6, we have

$$
\alpha(T u)=\min _{0 \leq x \leq 1}|T u(x)| \geq N \max _{0 \leq x \leq 1}|T u(x)|=N \theta(T u)>N \frac{b}{N}=b
$$

so condition $\left(\mathrm{S}_{2}\right)$ holds.

Step 4. We will show that condition $\left(S_{3}\right)$ of Theorem 3.1 is also satisfied. Obviously, $\psi(0)=0<a$, and we have $0 \notin R(\psi, \gamma, a, d)$. Assume that $u \in R(\psi, \gamma, a, d)$ with $\psi(u)=a$. Then, from condition $\left(B_{3}\right)$, we have

$$
\begin{aligned}
\psi(T u) & =\max _{0 \leq x \leq 1}|T u(x)|=(T u)\left(\frac{1}{2}\right) \\
& =\int_{0}^{1}\left(H(s)+G\left(\frac{1}{2}, s\right)\right) f\left(s, u(s), u^{\prime}(s)\right) d s \\
& <\frac{a}{L} \int_{0}^{1}\left(H(s)+G\left(\frac{1}{2}, s\right)\right) d s=a .
\end{aligned}
$$


It proves that condition $\left(S_{3}\right)$ holds. All conditions of Theorem 3.1 are satisfied and we assert that BVP (1.1) has at least three concave symmetric positive solutions $u_{1}, u_{2}, u_{3} \in P$ such that

$$
\begin{aligned}
& \max _{0 \leq x \leq 1}\left|u_{i}^{\prime}(x)\right| \leq d \quad \text { for } i=1,2,3 ; \quad \max _{0 \leq x \leq 1}\left|u_{1}(x)\right|<a, \\
& \max _{0 \leq x \leq 1}\left|u_{2}(x)\right|>a \quad \text { with } \min _{0 \leq x \leq 1}\left|u_{2}(x)\right|<b, \quad \min _{0 \leq x \leq 1}\left|u_{3}(x)\right|>b .
\end{aligned}
$$

Therefore, our proof is complete.

\section{Example}

Example 4.1 We consider the following three-point second-order BVP with integral boundary conditions:

$$
\left\{\begin{array}{l}
u^{\prime \prime}(x)+f\left(x, u(x), u^{\prime}(x)\right)=0, \quad x \in(0,1) \\
u(0)=u(1)=\frac{1}{8} \int_{0}^{\frac{1}{4}} u(s) d s
\end{array}\right.
$$

where

$$
f\left(x, u, u^{\prime}\right)= \begin{cases}x(1-x)+u^{11}+\frac{\sqrt{\left|u^{\prime}\right|}}{1,025}, & 0 \leq u \leq 2, \\ x(1-x)+2,048+\frac{\sqrt{\left|u^{\prime}\right|}}{1,025}, & u \geq 2 .\end{cases}
$$

We can see from (4.1) that $\alpha=\frac{1}{8}, \eta=\frac{1}{4}$. Then $M=\frac{16}{31}, N=\frac{1}{31}, L=\frac{377}{2,976}, \delta=\frac{5}{2,976}$. We choose $a=1, b=2, d=1,025$. Clearly $0<a<b \leq 2 d \delta$. Moreover, $f$ satisfies (H1).

(B) $\quad f(x, u, v) \leq \frac{1}{4}+2,048+\frac{\sqrt{1,025}}{1,025}<2 d=2,050$

$$
\text { for }(x, u, v) \in[0,1] \times\left[0, \frac{16,400}{31}\right] \times[-1,025,1,025] \text {; }
$$

(B) $f(x, u, v)>2,048>\frac{b}{\delta}=1,190.4 \quad$ for $(x, u, v) \in[0,1] \times[2,62] \times[-1,025,1,025]$;

(B) $f(x, u, v)<\frac{1}{4}+1+\frac{\sqrt{1,025}}{1,025}<\frac{a}{L} \approx 7.9$

$$
\text { for }(x, u, v) \in[0,1] \times[0,1] \times[-1,025,1,025] \text {. }
$$

So, by Theorem 3.2 we find that BVP (4.1) has at least three concave symmetric positive solutions $u_{1}, u_{2}, u_{3}$ such that

$$
\begin{aligned}
& \max _{0 \leq x \leq 1}\left|u_{i}^{\prime}(x)\right| \leq 1,025 \quad \text { for } i=1,2,3 ; \quad \max _{0 \leq x \leq 1}\left|u_{1}(x)\right|<1, \\
& \max _{0 \leq x \leq 1}\left|u_{2}(x)\right|>1 \quad \text { with } \min _{0 \leq x \leq 1}\left|u_{2}(x)\right|<2, \quad \min _{0 \leq x \leq 1}\left|u_{3}(x)\right|>2 .
\end{aligned}
$$


Authors' contributions

All authors contributed equally to the manuscript and typed, read, and approved the final manuscript.

\section{Author details}

'Department of Mathematics, Faculty of Science, Anadolu University, Eskisehir, 26470, Turkey. ${ }^{2}$ Department of Mathematics, Ege University, Bornova, Izmir, 35100, Turkey.

Received: 10 September 2014 Accepted: 17 November 2014 Published: 09 Dec 2014

\section{References}

1. Sun, $Y$, Zhang, $X$ : Existence of symmetric positive solutions for an $m$-point boundary value problem. Bound. Value Probl. 2007, Article ID 79090 (2007)

2. Kosmatov, N: Symmetric solutions of a multi-point boundary value problem. J. Math. Anal. Appl. 309, 25-36 (2005)

3. Zhao, J, Miao, C, Ge, W, Zhang, J: Multiple symmetric positive solutions to a new kind of four point boundary value problem. Nonlinear Anal. 71, 9-18 (2009)

4. Hao, F: Existence of symmetric positive solutions for $m$-point boundary value problems for second-order dynamic equations on time scales. Math. Theory Appl. 28, 65-68 (2008)

5. Pang, $\mathrm{H}$, Tong, Y: Symmetric positive solutions to a second-order boundary value problem with integral boundary conditions. Bound. Value Probl. 2013, 150 (2013). doi:10.1186/1687-2770-2013-150

6. Il'in, VA, Moiseev, El: Nonlocal boundary value problem of the second kind for a Sturm-Liouville operator. Differ. Equ. 23, 979-987 (1987)

7. Gupta, CP: A generalized multi-point boundary value problem for second order ordinary differential equations. Appl. Math. Comput. 89, 133-146 (1998)

8. Feng, W: On an m-point boundary value problem. Nonlinear Anal. 30, 5369-5374 (1997)

9. Sun, B, Ge, W, Zhao, D: Three positive solutions for multipoint one-dimensional $p$-Laplacian boundary value problems with dependence on the first order derivative. Math. Comput. Model. 45, 1170-1178 (2007)

10. Jankowski, T: Differential equations with integral boundary conditions. J. Comput. Appl. Math. 147, 1-8 (2002)

11. Boucherif, A: Second-order boundary value problems with integral boundary conditions. Nonlinear Anal. 70, 364-371 (2009)

12. Tariboon, J, Sitthiwirattham, T: Positive solutions of a nonlinear three-point integral boundary value problem. Bound. Value Probl. 2010, Article ID 519210 (2010). doi:10.1155/2010/519210

13. Avery, RI, Peterson, AC: Three positive fixed points of nonlinear operators on ordered Banach spaces. Comput. Math. Appl. 42, 313-322 (2001)

10.1186/1687-1847-2014-313

Cite this article as: Akcan and Hamal: Existence of concave symmetric positive solutions for a three-point boundary value problems. Advances in Difference Equations 2014, 2014:313

\section{Submit your manuscript to a SpringerOpen ${ }^{\circ}$ journal and benefit from:}

- Convenient online submission

- Rigorous peer review

- Immediate publication on acceptance

- Open access: articles freely available online

- High visibility within the field

- Retaining the copyright to your article 